\title{
A New Etiologic Factor for Adnexal Torsion: Congenital Omental Fenestrum
}

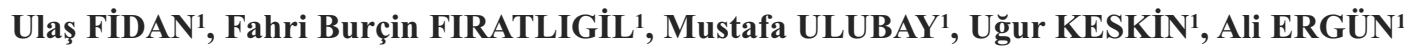 \\ Ankara, Turkey
}

\begin{abstract}
Torsion seen in ovary, fallopian tube or in both organs is one of the gynecologic emergency pathologies in patients suffering from acute lower abdominal pain, and it constitutes approximately $2.7 \%$ of gynecologic emergencies. Delays in diagnosis and treatment can cause ischemic damage, in that way can cause the risk of ovary, fallopian tube or both organs loss. Thus, it can result in fertility problems in people who are desiring future fertility.

Although the etiology is not totally known, it has been thought that torsion risk has been increasing in benign cystic teratomas and ovarian tumors. It can be also seen in congenital situations such as vitellointestinal duct anomaly and people who have undergone pelvic surgery.

In this article, a case for adnexal torsion developed in congenital omental fenestrum which is considered as a new factor for torsion etiology, was explained.
\end{abstract}

Keywords: Adnexal torsion, Congenital omental fenestrum

Gynecol Obstet Reprod Med 2016;22:45-46

\section{Introduction}

Torsion seen in ovary, fallopian tube or in both organs is one of the gynecologic emergency pathologies in patients suffering from acute lower abdominal pain ${ }^{1}$, and it constitutes approximately $2.7 \%$ of gynaecologic emergencies. ${ }^{2}$ Not only the adnexal torsion cases are seen in patients in their reproductive ages, but also rarely in premenarchal and postmenopausal ages. $^{3}$

The cases are generally faced when ovary, fallopian tube or both organs together rotate as well their own vascular pedicle around broad ligament at least one tour. Although the etiology is not totally known, it has been thought that torsion risk has been increasing in benign cystic teratomas and ovarian tumors. ${ }^{2}$ In the case of torsion, benign adnexal lesions are riskier than malignant lesions, because in malignant lesions, there would be adhesions between ovary and neighboring tissues and this can decrease the possibility of torsion cases. ${ }^{4}$ Additionally, it is thought that undergoing pelvic surgery procedures can also increase the risk of adnexal torsion. ${ }^{5}$

Quick diagnosis and treatment are extremely important in

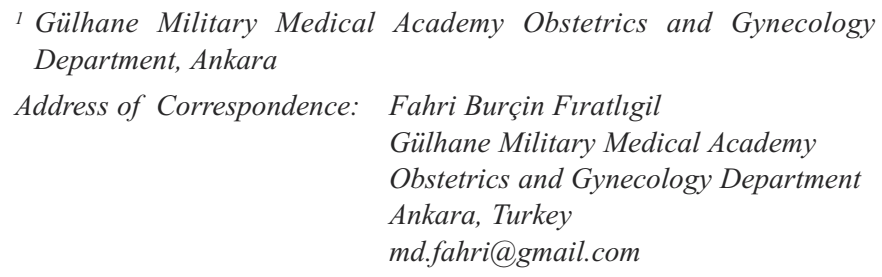

Submitted for Publication:

30. 09. 2014

Accepted for Publication: adnexal torsion cases. Because, delays in diagnosis and treatment can cause ischemic damage, in that way can cause the risk of ovary, fallopian tube or both organs loss. Thus, it can result in fertility loss in people who are desiring future fertility. ${ }^{1}$

In this article, a case for adnexal torsion developed in congenital omental fenestrum in the patient who did not have any risk factor, was explained.

\section{Case Report}

The nulligravida patient with the age of 29 was consulted to our clinics from emergency department with her complains of increasing left inguinal pain, nausea and vomitting.

While there was no gynecopathology or gynecologic operation in her gynecologic history, rebound and tenderness were observed in left lower quadrant examination. Cervical motion pain and left adnexal tenderness were observed in bimanual examination. On pelvic ultrasound examination enlarged-hypoechogenic left adnexal structures; low velocity peripheral and intra-ovarian venous flow in doppler sonography were detected. In laboratory investigations; white blood cell count was 10.400 cells per microliter (normal range: 4.100-11.2000 cells per microliter), $\beta$-human chorionic gonadotropin ( $\beta$ $\mathrm{hCG}$ ) was negative, urinalysis and biochemical parameters were also normal.

Initially, diagnostic laparoscopy was performed with a preliminary diagnosis of adnexal torsion. When pelvic cavity was visualised, two times rotated left adnexal structures were seen in omental defect (Figure 1). The adnexal structures which 
were not considered as necrotic, were detorsioned by being taken from omental defect. After this procedure, blood supply of ovary and fallopian tube were observed as normal and there was no sign of any cystic or bulky lesion. When omentum was examined intraoperatively, there was a defect whose dimensions were measured as approximately $3 \mathrm{~cm} \times 5 \mathrm{~cm}$. Because the patient did not have any prior abdominal/pelvic surgeries or other intraabdominal diseases in her past medical history, this defect was considered as a congenital fenestrum. The defect was excised by laparoscopic cutting forceps at the lateral side, so that the defect had been eradicated.

The patient was discharged without any complications or complaints at the first post operative day.

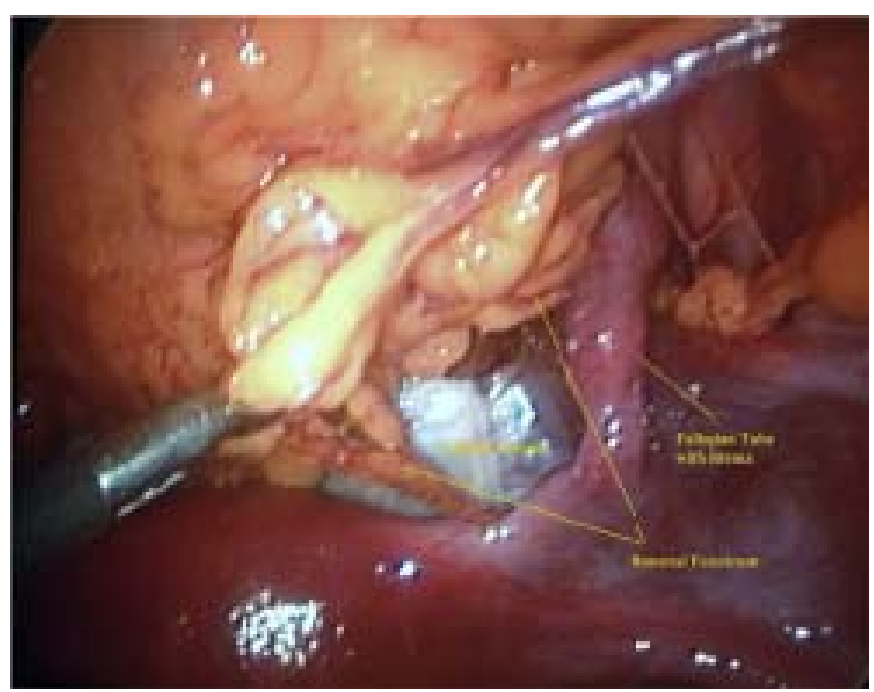

Figure 1: Torsioned adnexal structures in the omental fenestrum

\section{Discussion}

Although the etiology of adnexal torsion is not clearly known, it has critical place in gynecological emergencies as quick diagnosis and treatment are needed for this disease.

As mentioned before, benign ovarian cysts, especially teratomas are taking place in etiology. Beside this, undergoing pelvic surgical procedures can cause future adnexal torsion. It has been previously demonstrated that adnexal torsion case can also be generated due to vitello- intestinal duct anomaly and it considered as a new factor for the torsion etiology in congenital anomalies. ${ }^{6}$

In our case, adnexal structures were torsioned in congenital fenestrum which was in omentum. Although it was thought that adnexa located in omental defect can cause torsion since omentum is an active organ; the adnexal torsion case seen in omental fenestrum has not been revealed in any literature so far. In our case, there were no history of undergoing prior pelvic surgery or gynecopathological diseases. That is why, this congenital omental fenestrum could play the key role for torsion.

In conclusion, adnexal parts can cause torsion by locating in congenital omental fenestrum and this can be placed in medical literature as a rare case. Thus, omentum should be carefully examined in patients who will have pelvic surgery and any defect development should be avoided. In addition, as in our case, in torsion cases having no risk factor, omental defect or fenestrum should be considered.

\section{References}

1. Huchon C, Fauconnier A. Adnexal torsion: a literature review. Eur J Obstet Gynecol Reprod Biol 2010;150(1):8 12 .

2. Oelsner G, Shashar D. Adnexal Torsion. Clin Obstet Gynecol 2006 Sep;49(3):459-63.

3. Houry D, Abbott JT. Ovarian torsion:a fifteen-year review. Ann Emerg Med 2001;38:156-9.

4. Sommerville M, Grimes DA, Koonings PP, Campbell K. Ovarian neoplasms and the risk of adnexal torsion. Am J Obstet Gynecol 1991;164(2):577-8.

5. Becker JH, de Graaff J, Vos CM. Torsion of the ovary: a known but frequently missed diagnosis. Eur J Emerg Med 2009;16(3):124-6.

6. Lin W, Xiaohui L, Rutie Y, Donghao L. A rare confounder in a case of ovarian torsion: letters to the editor. Eur $\mathrm{J}$ Obstet Gynecol Reprod Biol 2012;163(1):117-22. 